\title{
BMJ Open Ablation with irreversible electroporation in patients with advanced perihilar cholangiocarcinoma (ALPACA): a multicentre phase I/II feasibility study protocol
}

\author{
Robert J S Coelen, ${ }^{1}$ Jantien A Vogel, ${ }^{1}$ Laurien G P H Vroomen, ${ }^{2}$ Eva Roos, ${ }^{1}$ \\ Olivier R C Busch, ${ }^{1}$ Otto M van Delden, ${ }^{3}$ Foke van Delft, ${ }^{4}$ Michal Heger, ${ }^{1}$ \\ Jeanin E van Hooft, ${ }^{5}$ Geert Kazemier, ${ }^{6}$ Heinz-Josef Klümpen, ${ }^{7}$ Krijn P van Lienden, ${ }^{3}$ \\ Erik A J Rauws, ${ }^{5}$ Hester J Scheffer, ${ }^{2}$ Henk M Verheul, ${ }^{8}$ Jan de Vries, ${ }^{2}$ \\ Johanna W Wilmink, ${ }^{7}$ Barbara M Zonderhuis, ${ }^{6}$ Marc G Besselink, ${ }^{1}$ \\ Thomas M van Gulik, ${ }^{2}$ Martijn R Meijerink ${ }^{2}$
}

To cite: Coelen RJS, Vogel JA, Vroomen LGPH, et al. Ablation with irreversible electroporation in patients with advanced perihilar cholangiocarcinoma (ALPACA): a multicentre phase I/II feasibility study protocol. BMJ Open 2017;7:e015810. doi:10.1136/ bmjopen-2016-015810

- Prepublication history and additional material for this paper are available online. To view these files please visit the journal online (http://dx.doi. org/10.1136/bmjopen-2016015810).

RJSC and JAV contributed equally.

Received 3 January 2017 Revised 8 June 2017 Accepted 9 June 2017

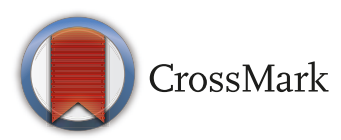

For numbered affiliations see end of article.

Correspondence to

Robert J S Coelen;

r.j.coelen@amc.nl

\section{ABSTRACT}

Introduction The majority of patients with perihilar cholangiocarcinoma (PHC) has locally advanced disease or distant lymph node metastases on presentation or exploratory laparotomy, which makes them not eligible for resection. As the prognosis of patients with locally advanced PHC or lymph node metastases in the palliative setting is significantly better compared with patients with organ metastases, ablative therapies may be beneficial. Unfortunately, current ablative options are limited. Photodynamic therapy causes skin phototoxicity and thermal ablative methods, such as stereotactic body radiation therapy and radiofrequency ablation, which are affected by a heat/cold-sink effect when tumours are located close to vascular structures, such as the liver hilum. These limitations may be overcome by irreversible electroporation (IRE), a relatively new ablative method that is currently being studied in several other soft tissue tumours, such as hepatic and pancreatic tumours. Methods and analysis In this multicentre phase I/II safety and feasibility study, 20 patients with unresectable PHC due to vascular or distant lymph node involvement will undergo IRE. Ten patients who present with unresectable PHC will undergo CT-guided percutaneous IRE, whereas ultrasound-guided IRE will be performed in 10 patients with unresectable tumours detected at exploratory laparotomy. The primary outcome is the total number of clinically relevant complications (Common Terminology Criteria for Adverse Events, score of $\geq 3$ ) within 90 days. Secondary outcomes include quality of life, tumour response, metal stent patency and survival. Follow-up will be 2 years.

Ethics and dissemination The protocol has been approved by the local ethics committees. Data and results will be submitted to a peer-reviewed journal.

Conclusion The Ablation with irreversible eLectroportation in Patients with Advanced perihilar CholangiocarcinomA (ALPACA) study is designed to assess the feasibility of IRE for advanced PHC. The main purpose is to inform whether
Strengths and limitations of this study

Both the open and percutaneous approach of irreversible electroporation for perihilar cholangiocarcinoma are prospectively investigated.

- Quality of life of participating patients will be closely observed using validated questionnaires.

- Strict eligibility criteria may slow the accrual of study participants.

- The feasibility study design with consequent relative small sample size does not allow for accurate survival analysis.

a follow-up trial to evaluate safety and effectiveness in a larger cohort would be feasible.

\section{INTRODUCTION}

Perihilar cholangiocarcinoma (PHC) is a rare biliary malignancy originating at or near the hepatic duct confluence with an annual incidence of approximately 200 patients in the Netherlands. ${ }^{12}$ The typical location in the liver hilum causes biliary obstruction with concomitant jaundice. Surgical resection of PHC consists of a combined extrahepatic bile duct resection and partial liver resection, and offers the best chance for long-term survival, with a reported median overall survival (OS) of 19-40 months and 5-year survival rates of $13 \%-40 \% .^{3-5}$ Unfortunately, only a subset of patients are eligible to undergo surgical resection, as almost $50 \%$ of patients already present with unresectable disease, and of the patients undergoing exploratory laparotomy 
approximately $40 \%$ are found to have locally advanced or metastasised disease. ${ }^{6}$ Of all patients with ultimately unresectable disease, approximately $50 \%$ of tumours are considered locally advanced because of vascular involvement without the possibility of reconstruction or extensive biliary involvement. ${ }^{67}$

Systemic chemotherapy is the standard treatment for patients who are ineligible for curative resection or liver transplantation. The preferred regimen consists of a combination of gemcitabine and cisplatin and results in a median progression-free survival (PFS) of 8 months and a median OS of 12 months. ${ }^{8}$ Most patients eventually die of cholangitis, sepsis or liver failure, despite biliary stenting to relieve the cholestasis. Although the overall prognosis of unresectable PHC is poor, the median OS for patients with locally advanced PHC or lymph node metastases beyond the hepatoduodenal ligament is significantly longer (14-16 months) compared with patients with organ metastases (3-5 months). ${ }^{6}$ Long-term survival of up to 36 months has even been reported in some of these patients. This particular subgroup of patients with PHC may benefit from ablative therapies that counteract tumour growth and potentially improve biliary stent patency and survival. ${ }^{9}$

Several ablative therapies have been investigated for the treatment of advanced PHC, including stereotactic body radiation therapy (SBRT), ${ }^{10} 11$ photodynamic therapy $(\mathrm{PDT})^{12}{ }^{13}$ and endoscopic biliary radiofrequency ablation (RFA). ${ }^{14} 15$ These modalities show somewhat promising results but also suffer from major limitations. In PDT, severe skin phototoxicity due to the use of slowly degradable photosensitisers is reported. ${ }^{12}$ Furthermore, thermal ablative modalities (ie, RFA) are limited by thermal damage to surrounding structures and a so-called heat/cold-sink effect. The latter phenomenon is mediated by blood flow in surrounding vessels, creating an area where optimal temperatures are not reached, leaving viable cancer tissue in situ. ${ }^{16}$ Due to the typical location of PHC in the liver hilum near the portal vein and hepatic arteries, this is particularly challenging.

Irreversible electroporation (IRE) is an image-guided ablation technique based on creating short-pulsed highvoltage current fields. The electrical pulses permeabilise the lipid bilayer of the cell membrane, hereby disrupting intracellular homeostasis and inducing apoptosis. ${ }^{17-19}$ Because of the non-thermal mechanism, the effect of IRE is not counteracted by a heat/cold-sink effect and causes less damage to surrounding vital structures. In one clinical study including 101 patients, the effect of IRE on vessel patency in close proximity to the ablation zone was evaluated and showed that 151 of 158 major vessels were patent, whereas only 7 vessels exhibited thrombosis or mild narrowing at follow-up..$^{20}$ In another study, it was observed that tumours in close proximity to major bile ducts could be safely treated with IRE, as 26 of 28 evaluated bile ducts were patent 1 month after treatment (one occlusion occurred). ${ }^{21}$ Long-term patency of large hilar bile ducts has also been reported in a porcine model, especially when electrodes were positioned more than $2 \mathrm{~mm}$ away from the bile ducts. ${ }^{22}$

Evidence for the use of IRE in PHC is limited to one case report. ${ }^{23}$ However, several clinical studies have investigated the safety and effectiveness of IRE in hepatic and pancreatic tumours. IRE was shown to be relatively safe and feasible in locally advanced tumours in proximity of vital structures. ${ }^{24-26}$ In locally advanced pancreatic cancer, IRE-related complications have been reported in $13 \%$ of patients with $2 \%$ mortality, whereas IRE-related complications in hepatic tumours occurred in $16 \% .{ }^{27}{ }^{28}$ Complete response of hepatic tumours has been reported in $67 \%-100 \% .{ }^{28}$ The percutaneous IRE approach, using CT, has been reported to be safe, thereby making it a minimally invasive alternative to open IRE during surgical exploration. ${ }^{29-31}$ Although the short-term safety profile of IRE has been extensively documented, long-term results are awaited. Potentially improved survival has been reported in a selected group of patients with locally advanced pancreatic cancer. ${ }^{26} 32$

Given the lack of evidence on the use of IRE in the treatment of advanced PHC, the current study was designed to gather information regarding the safety and feasibility of IRE in these patients prior to palliative chemotherapy.

\section{METHODS AND ANALYSIS Design}

The Ablation with irreversible eLectroportation in Patients with Advanced perihilar CholangiocarcinomA (ALPACA) study is a multicentre, phase I/II safety and feasibility study of the use of IRE treatment with the NanoKnife system (AngioDynamics, Amsterdam ZuidOost, the Netherlands) in 20 patients with advanced PHC. In this study, advanced PHC is defined as PHC with vascular involvement or distant lymph node metastases, precluding a resection. Two groups will be distinguished in the study. Patients who present with advanced PHC or have evidence of advanced disease during preoperative work-up will undergo percutaneous CT-guided IRE. Patients who present with potentially resectable PHC that appears to be locally advanced or metastasised to distant lymph nodes at exploratory laparotomy will undergo ultrasound (US)-guided IRE during the same surgical exploration session. Criteria for advanced PHC are summarised in online supplementary additional file 1 . The study is conducted at the Academic Medical Center and VU University Medical Center, which both are tertiary referral centres for patients with PHC in Amsterdam, the Netherlands.

\section{Study objectives}

The objective of this study is to assess the safety and feasibility of IRE for the treatment of advanced PHC with vascular or lymph node involvement prior to palliative chemotherapy. The main purpose of this feasibility study is to inform whether a follow-up trial to evaluate safety and effectiveness in a larger cohort would be feasible. 


\section{Primary study outcome}

The primary outcome is the total number of clinically relevant complications within 90 days post-IRE, defined as complications requiring reintervention, prolonged hospital stay, intensive care admission, readmission or complications leading to mortality. These complications are summarised by a Common Terminology Criteria for Adverse Events (CTCAE, V.4.0) score of 3 or higher. Definitions of specific postprocedural complications are provided in online supplementary additional file 2 .

\section{Secondary study outcomes}

- Technically successful IRE procedure (ability to correctly place the IRE needles and complete the procedure)

- Intraprocedural complications related to IRE (cardiac arrhythmias, perforation of organs or vascular structures)

$\checkmark$ Duration of hospital stay

-Quality of life at baseline (outpatient clinic), 6 weeks, 3 months and 6 months after IRE; assessments consists of the European Organisation for Research and Treatment of Cancer Quality of Life Questionnaire 30 (EORTC QLQ-C30), the EORTC QOL module for cholangiocarcinoma and gallbladder cancer (EORTC QLQ-BIL21) and the EuroQol 5D

- Tumour response on CT imaging (Response Evaluation Criteria in Solid Tumours (RECIST) 1.1 and criteria in table 1) and blood biomarker response (carbohydrate antigen 19-9 (CA 19-9)) at 6 weeks and 6, 12 and 24 months after IRE

- Long-term vascular and biliary complications (thrombosis, bile duct stenosis) as assessed on follow-up CT imaging

- Time interval between IRE and start of palliative chemotherapy

Duration of palliative chemotherapy (number of cycles)

- Metal stent patency (time between stent insertion and replacement)

- Survival (1-year and 2-year PFS and OS). PFS is defined as the number of months between the IRE procedure and the time of first evidence of progressive disease as defined by the RECIST criteria and proposed criteria in table 1 . OS is defined as the number of months between the IRE procedure and time of death or last follow-up.

\begin{tabular}{|c|c|c|c|c|}
\hline & $\begin{array}{l}\text { Complete } \\
\text { response (CR) }\end{array}$ & $\begin{array}{l}\text { Partial } \\
\text { response (PR) }\end{array}$ & $\begin{array}{l}\text { Stable } \\
\text { disease (SD) }\end{array}$ & $\begin{array}{l}\text { Progressive } \\
\text { disease (PD) }\end{array}$ \\
\hline \multicolumn{5}{|l|}{$\begin{array}{l}\text { Major criteria } \\
\text { One sufficient for PD }\end{array}$} \\
\hline $\begin{array}{l}\text { Tumour size* } \\
\text { Longest diameter on axial or coronal plane of the solid } \\
\text { enhancing part of the tumour }\end{array}$ & $\begin{array}{l}\text { No residual } \\
\text { solid enhancing } \\
\text { tumour }\end{array}$ & $\begin{array}{l}\text { Decrease } \\
>30 \%\end{array}$ & $\begin{array}{l}\text { Decrease } \\
\leq 30 \% \text { or } \\
\text { increase } \leq 20 \%\end{array}$ & $\begin{array}{l}\text { Increase } \\
>20 \%\end{array}$ \\
\hline $\begin{array}{l}\text { Metastases } \\
\text { New onset, pathology-proven lymph node metastases; } \\
\text { cytology-proven ascites (peritonitis carcinomatosa); distant } \\
\text { non-nodal metastases }\end{array}$ & No & No & No & Yes \\
\hline
\end{tabular}

Minor criteria

$\geq 2$ needed for $P D$ in absence of major criteria

\begin{tabular}{lc} 
New-onset vessel narrowing* & No \\
$\begin{array}{l}\text { 50\% diameter reduction of portal vein, hepatic artery or } \\
\text { other major vessels in absence of thrombosis }\end{array}$ & No \\
$\begin{array}{l}\text { New-onset biliary obstruction* } \\
\text { Other cause than biliary stent clogging or migration }\end{array}$ & No \\
$\begin{array}{l}\text { New lymph nodes* } \\
>15 m m \text { short axis diameter in transversal plane }\end{array}$ & No \\
$\begin{array}{l}\text { New-onset ascites* } \\
\text { Cytology negative (otherwise PD); exudate; serum ascites } \\
\text { albumin gradient }<1.1 ; \text { CA } 19-9 \text { (ascites) }>74 \mathrm{U} / \mathrm{mL}\end{array}$ & \\
$\begin{array}{l}\text { CA } 19-9 \text { (plasma) increase }>100 \% \text { (and }>74 \mathrm{U} / \mathrm{mL}) t \\
\text { Without signs for pancreatitis or biliary obstruction }\end{array}$ & No \\
\hline
\end{tabular}

Criteria will be compared with RECIST 1.1 as prognosticators for overall survival for validation. Follow-up CT scans are performed at 6 weeks post-IRE and 6, 12 and 24 months post-IRE.

${ }^{*}$ All criteria are compared with the first follow-up scan 6 weeks post-IRE.

†Compared with pretreatment value. CA 19-9 rise only significant if latest value is at least $2 \times$ the upper limit of normal $(2 \times 37 \mathrm{U} / \mathrm{mL})$.

CA 19-9, carbohydrate antigen 19-9; IRE, irreversible electroporation; PHC, perihilar cholangiocarcinoma; RECIST 1.1, Response Evaluation Criteria in Solid Tumours 1.1. 


\section{Hypotheses}

Our primary hypothesis is that IRE will add approximately $20 \%-25 \%$ clinically relevant complications (CTCAE $\geq 3$ ) to the current $40 \%$ complication rate associated with biliary drainage in the palliative management of PHC. ${ }^{33}$ An incidence of $60 \%$ complications within 90 days post-IRE will be considered as the acceptable upper limit. This will be related to the observed impact on survival. Our secondary hypothesis is that the patency rate of metal stents will be prolonged because of local tumour control. Furthermore, we hypothesise that median OS is increased by 3 months in patients undergoing IRE. Both the metal stent patency rate and OS of patients in this study will be compared with patients who were eligible for study participation but preferred standard palliative treatment. When necessary, study data may also be compared with a historical cohort of patients treated in our centre.

\section{Study population}

The study population consists of 20 adults who are diagnosed with either advanced PHC based on imaging or staging laparoscopy, or potentially resectable PHC but ultimately advanced disease during exploratory laparotomy. Patients will be recruited at the outpatient clinic. In case of a planned exploratory laparotomy, patients will be asked to participate in the study in case they appear to have locally advanced or lymph node metastasised PHC at laparotomy. Patients with intrahepatic or extrahepatic organ metastases are not eligible for study participation. Inclusion and exclusion criteria are listed in table 2.

Patients who are candidates for percutaneous CT-guided IRE or US-guided open IRE will be discussed upfront by the ALPACA expert panel. This dedicated panel consists of three interventional radiologists ( $\mathrm{KPvL}, \mathrm{OMvD}, \mathrm{MRM})$, three gastroenterologists (JEvH, EAJR, FvD) and four surgeons (MGB, ORCB, GK, TMvG) .

\section{Study outline}

The general outline of study procedures is presented in figure 1. Patients with potentially resectable PHC undergo the standard preoperative work-up including laboratory testing, CT or MRI, biliary drainage, liver function tests and portal vein embolisation (when indicated). Patients who present with unresectable tumours will undergo CT imaging, laboratory testing and biliary drainage as part of the work-up for percutaneous IRE.

\section{Preprocedural biliary drainage}

Biliary drainage for malignant hilar strictures or masses is a complex procedure requiring considerable skill and experience. In the present study, the exact approach of biliary drainage (ie, endoscopically or percutaneously, specified liver segments, unilateral or bilateral) prior to percutaneous IRE is decided for every patient individually as it depends on biliary anatomy, vascular involvement, lobar atrophy and any cause of previous drainage

Table 2 Inclusion and exclusion criteria

\begin{tabular}{|c|c|}
\hline Inclusion criteria & Exclusion criteria \\
\hline $\begin{array}{l}\text { Age } \geq 18 \text { years } \\
\text { WHO performance status } \leq 2 \\
\text { Advanced } \mathrm{PHC}^{*} \\
\text { - Excessive vascular involvement } \\
\text { precluding } \mathrm{R} 0 \text { or } \mathrm{R} 1 \text { resection } \\
\text { - Lymph node metastases beyond the } \\
\text { hepatoduodenal ligament (common } \\
\text { hepatic artery, periaortic, pericaval, } \\
\text { superior mesenteric artery, coeliac } \\
\text { artery) }\end{array}$ & $\begin{array}{l}\text { Resectable PHC on exploratory laparotomy } \\
\text { Locally advanced PHC eligible for liver transplantation } † \\
\text {-5 cm tumour extension along the common hepatic duct or common bile duct } \\
\text { biopsy, staging laparoscopy or intraoperative frozen section } \\
\text { Lymph node metastases beyond N2 stations (eg, inguinal, mediastinal) } \\
\text { Locoregional recurrence of PHC } \\
\text { History of cardiac arrhythmias (sinus tachycardia (BPM }>100 \text { ), sick sinus } \\
\text { syndrome, sinoatrial exit block, AV block, sinus node re-entry, presence of } \\
\text { pacemaker or defibrillator) } \\
\text { Recent history of myocardial infarction (<6 months) } \\
\text { Uncontrolled hypertension (blood pressure must be } \leq 160 / 95 \text { mm Hg at the time of } \\
\text { screening on a stable antihypertensive regimen } \\
\text { Uncontrolled infections (>grade } 2 \text { CTCAE V.4) } \\
\text { Epilepsy } \\
\text { Partial or complete portal vein thrombosis } \\
\text { Both narrowing (sclerosis) of the portal vein and a reduced diameter of either the } \\
\text { common hepatic artery, coeliac trunk or superior mesenteric artery of }>50 \% \\
\text { Any condition that is unstable or that could jeopardise the safety of the subject } \\
\text { and their compliance in the study }\end{array}$ \\
\hline
\end{tabular}

${ }^{*}$ Detailed criteria in online supplementary additional file 2. Diagnosis of PHC or lymph node metastases will be confirmed with endoscopic brush, percutaneous or laparoscopic biopsy, whichever is suitable. Vascular or lymph node involvement on laparotomy will be confirmed with intraoperative frozen section.

†Detailed criteria in online supplementary additional file 4.

AV, atrioventricular; BPM, beats per minute; CTCAE, Common Terminology Criteria for Adverse Events; PHC, perihilar cholangiocarcinoma. 


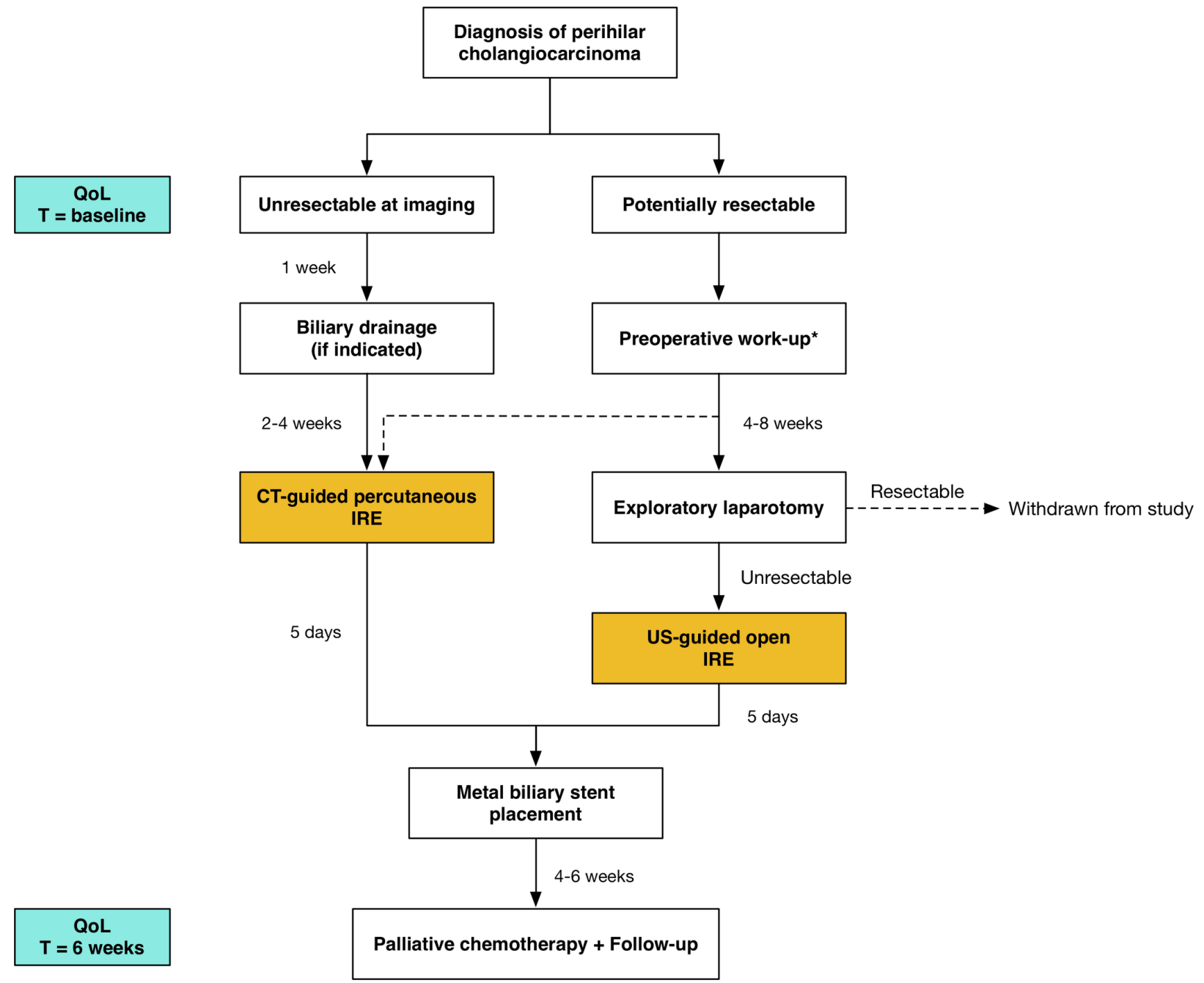

Figure 1 Flow chart of study procedures and timing of interventions. *May include biliary drainage, staging laparoscopy, liver function assessment, portal vein embolisation and preoperative radiotherapy. IRE, irreversible electroporation; QoL, quality of life; US, ultrasound.

failure at the referring hospital. Preferably, bilateral stenting is performed to protect the bile ducts during IRE. This decision is made by the ALPACA expert panel. All drainage procedures prior to IRE will be performed using plastic stents. A metal biliary stent (sometimes placed at the referring hospital) is not considered a contraindication for IRE as long as a no-touch technique is pursued (ie, IRE electrodes are not in contact with the metal stent). ${ }^{345}$ IRE will be performed when bilirubin levels have decreased below $50 \mu \mathrm{mol} / \mathrm{L}$, but this threshold is not an absolute contraindication for IRE, as obtaining adequate drainage may be difficult in some patients (eg, PHC arising from primary sclerosing cholangitis).

Candidates for open IRE who initially present with potentially resectable PHC will undergo preoperative biliary drainage as part of the standard preoperative workup. The optimal method (endoscopically or percutaneously) is decided at the multidisciplinary team meeting, based on biliary anatomy and the cause of previous drainage failure. ${ }^{36}$
Antibiotic prophylaxis

Antibiotic prophylaxis will be administered according to the local hospital's protocol within 1 hour prior to biliary drainage or IRE. In the Academic Medical Center, ceftriaxone (2000 $\mathrm{mg}$ once intravenously) plus gentamicin $(5 \mathrm{mg} / \mathrm{kg}$ once intravenously) are given. In the VU University Medical Center, ceftriaxone (2000 mg once intravenously) alone (first choice) or augmentin ( $1200 \mathrm{mg}$ every 6 hours intravenously) plus gentamicin ( $5 \mathrm{mg} / \mathrm{kg}$ once intravenously) (second choice) is given. The same antibiotics regimens will be used for the treatment of cholangitis.

\section{IRE procedure}

The IRE procedure will be performed under general anaesthesia and epidural analgesia (or preperitoneal wound catheters in the open IRE group) with complete muscle paralysis and under cardiac gating either in the operating room (US-guided open group) or in the interventional radiology suite (CT-guided percutaneous group). During the procedure, cardiac rhythm will be 
closely monitored and a defibrillator will be present at all times. All electrodes will be placed by trained interventional radiologists with extensive experience with the procedure in other solid tumours (eg, locally advanced pancreatic cancer).

Prior to percutaneous IRE, a pigtail catheter will be placed in the common hepatic artery in the angiography suite entering from the right common femoral artery, for the administration of small amounts of intra-arterial contrast during IRE. This allows repeated and real-time visualisation of the vessels adjacent to or encasing the tumour and the tumour enhancement pattern, thereby improving the safety and accuracy of electrode placement while reducing the total dose of contrast fluid administered. Next, the patient is transported to the CT scan. Procedures will be performed percutaneously under CT fluoroscopy guidance with transcatheter CT arteriography and/or ultrasonographic guidance. After correct patient positioning, a contrast-enhanced CT scan of the abdomen will be performed to confirm correct staging, exact tumour size measurement and for planning of electrode placement. US-guided open IRE will be performed during exploratory laparotomy when locally advanced PHC or distant lymph node metastases are found.

The NanoKnife IRE system (AngioDynamics) will be used in this study. The IRE is set up to produce 90-microsecond high-voltage (1500-3000 V) direct-current (25-45 A) electrical pulses. Typically, 90 pulses will be delivered in 9 sets of 10 pulses between paired unipolar electrodes, with an exposed tip of $2.0 \mathrm{~cm}$ (online supplementary additional file 3 ). The voltage setting for each electroporation will be determined by the distance between each pair of electrodes and will be aimed at $1500 \mathrm{~V} / \mathrm{cm}$, with the intent to generate at least $1000 \mathrm{~V}$ between electrodes. The electrodes will be placed in and around the tumour under CT guidance or US guidance according to the manufacturer's guidelines aiming at macroscopic complete ablation with a $5 \mathrm{~mm}$ margin, with interelectrode distances of $10-24 \mathrm{~mm}$ and a maximum angulation between electrodes of $15^{\circ}$. The predicted treatment zone will be automatically calculated using the NanoKnife generator software based on the exact position of all electrodes.

The number of probes used for ablation depends on tumour size and shape. For a two-dimensional ablation zone with a size of less than $30 \times 25 \times 10 \mathrm{~mm}$, two probes will be used (one active and one standard probe). When the shape of the ablation zone is more three-dimensional, with a maximum size of $30 \times 25 \times 20.5 \mathrm{~mm}$, three probes will be used (one active and two standard). For the ablation of a larger zone, that is, with a maximum size of $30 \times 25 \times 25 \mathrm{~mm}$, four probes will be used. Five probes will be used for tumours up to $30 \times 40 \times 30 \mathrm{~mm}$, and for larger tumours up to $30 \times 47 \times 32 \mathrm{~mm}$ six probes will be used. After satisfactory electrode placement, tumour ablation with the NanoKnife will be performed according to protocol under careful ECG monitoring. All pulses will be administered in the absolute refractory period with use of ECG synchronisation to avoid triggering of ventricular arrhythmias. If the lesion is larger or has a different shape than the area that one set of probes can cover (according to manufacturer's guidelines), multiple ablations will be performed, until the whole tumour area has been ablated. The generator will be programmed to stop delivery and recharge if the current flow exceeds $50 \mathrm{~A}$. Pull-backs will be performed if the target treatment zone is greater than $2 \mathrm{~cm}$ in the direction of needle placement, and treatment will be repeated to cover the entire target zone. The number of probes, number of probe replacements (per probe) due to unsatisfactory placement, number of pulses (for each pair of electrodes), interelectrode distance, pulse voltage $(\mathrm{V})$, achieved pulse current (Ampere), pulse length ( $\mu \mathrm{s})$ and pulse interval (ms) will be carefully recorded.

\section{Post-IRE treatment}

Patients will be monitored on the recovery (open group) and surgical ward (both groups), according to current medical practice. It may be necessary to perform additional drainage of undrained liver segments the day after open IRE. Patients undergoing percutaneous IRE will be discharged after 2-3 days (in the absence of complications). Endoscopic or percutaneous plastic drains will be exchanged for definitive metal stents (fully, partially or uncovered self-expanding metal stents) in all patients through an endoscopic or percutaneous approach preferably within 5 days after the IRE procedure. Bile ducts in atrophic liver lobes will preferably not be drained.

Patients will be treated with optimal palliative chemotherapy with gemcitabine and cisplatin. ${ }^{8}$ Palliative chemotherapy is preferably started within 6 weeks after IRE when patients have recovered from definitive metal biliary stent placement. The start of chemotherapy may be postponed in the event of IRE-related or biliary drainage-related complications. Gemcitabine $\left(1000 \mathrm{mg} / \mathrm{m}^{2}\right)$ and cisplatin $\left(25 \mathrm{mg} / \mathrm{m}^{2}\right)$ are administered on days 1 and 8 every 3 weeks with a total of 6-8 cycles. Chemotherapy will be discontinued in the event of tumour progression (based on RECIST 1.1 criteria and proposed criteria in table 1).

\section{Post-IRE follow-up}

Figure 2 presents a flow chart of the study follow-up. Six weeks and 6,12 and 24 months after treatment with IRE, an abdominal CT scan will be made in order to evaluate the effect of IRE (according to table 1). Blood samples will be drawn to measure tumour marker (CA 19-9) response. A CT scan 6 weeks post-IRE is considered the baseline scan for the start of palliative chemotherapy. When the time interval between this baseline scan and start of chemotherapy is prolonged with more than 4 weeks (ie, because of complications), a new baseline scan will be performed. Treatment response will be evaluated following every three cycles of chemotherapy. CT scans at 12 and 24 months post-IRE will be performed to evaluate the long-term tumour response following IRE. 


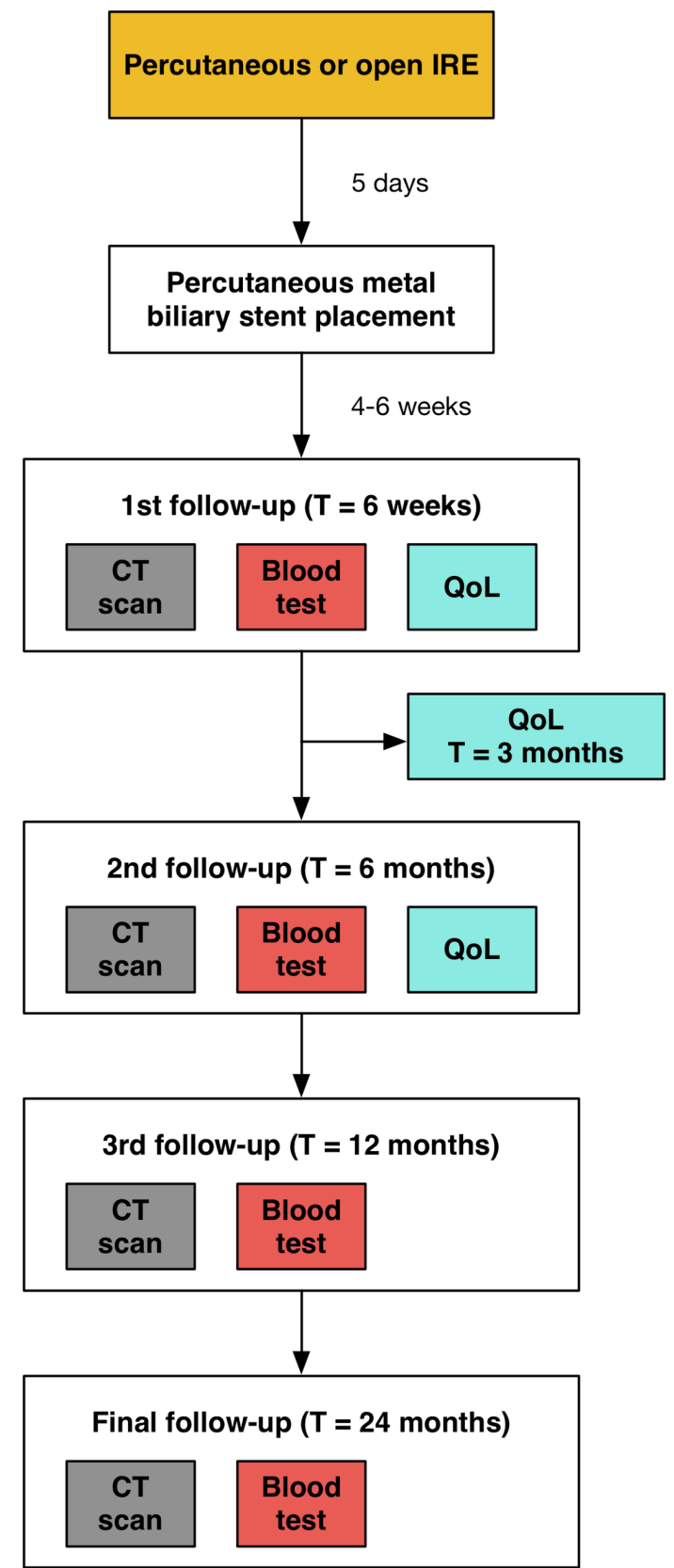

\section{Data analysis}

A blinded adjudication committee will assess the occurrence of the primary and secondary endpoints after the last patient has completed follow-up. The primary endpoint will be expressed as the total number of clinically relevant complications in the entire cohort as well as the treatment subgroups (percutaneous and open IRE). Also, the absolute complication risk will be calculated for the entire cohort and the treatment subgroups. Data will be presented as mean $\pm \mathrm{SD}$, and in case of non-normal distributions as median and IQR. Patient survival will be determined until 2 years after treatment. Patients still alive after 2 years and patients who are lost to follow-up will be censored. The data on OS and PFS will be presented as median survival in months with a CI of 95\%. Data on 1-year and 2-year survival will be presented as percentages.

\section{Sample size}

Considering the study's pilot design and lack of data on post-IRE complications in this patient group, a formal sample size calculation is not feasible. A cohort of 20 patients is suggested to be a reasonable size to investigate the safety and feasibility of the procedure. A range of 8-12 patients per treatment group is accepted while the total sample size remains 20 patients.

\section{ETHICS AND DISSEMINATION}

This study is conducted in accordance with the principles of the Declaration of Helsinki and the Dutch Medical 
Research Involving Human Subjects Act. The Institutional Review Board (IRB) of the Academic Medical Center in Amsterdam approved the protocol on 19 April 2016. Secondary approval was obtained from the local IRB at VU University Medical Center on 14 July 2016. The study has been registered with the Dutch Central Committee on Research Involving Human Subjects (NL56231.018.15) and The Netherlands National Trial Register (NTR5948, 4 July 2016). Informed consent will be obtained from each participating patient in oral and written form prior to any study procedures.

An independent data safety monitoring board (DSMB), consisting of three independent specialists (surgeon, interventional radiologist, medical oncologist), will examine the safety variables (eg, serious adverse events) in a non-blinded manner after completion of 90-day follow-up of the fifth and tenth study patient. The DSMB also assists and advises the trial steering committee so as to protect the validity and credibility of the study. Furthermore, annual non-blinded safety reports, including all serious adverse events per group, will be provided to the IRB. The study is monitored by the Clinical Research Unit of the Academic Medical Center.

Data will be presented at national and international conferences as soon as the primary endpoint has been reached for the final recruited patient. The results of the primary outcome (safety, complications within 90 days after IRE) will be published in a peer-reviewed journal. After completion of 2-year follow-up, results of the secondary survival outcomes will be presented and published as well. Data will be kept at the study sites for 15 years after trial completion, as in accordance with the Dutch Medical Research Involving Human Subjects Act.

\section{DISCUSSION}

The ALPACA study aims to assess the safety profile and feasibility of IRE for advanced PHC prior to palliative chemotherapy. The eventual results of this study will inform us whether a follow-up trial to evaluate safety and effectiveness in a larger cohort will be feasible. Previous studies in the field of hepato-pancreato-biliary (HPB) malignancies have reported acceptable short-term outcomes with the use of IRE in locally advanced hepatic and pancreatic tumours. IRE may potentially overcome the limitations of other modalities, such as skin phototoxicity in PDT, possible heat/cold-sink effect in RFA and the need for multiple fractions in SBRT. In the present study, IRE will not replace the current standard of treatment in patients with locally advanced PHC. Thus, patients meeting the criteria for liver transplantation will be referred to a transplant centre and all patients will be evaluated for systemic chemotherapy.

IRE may be associated with severe complications given the complex anatomy of the liver hilum and the close proximity of the hepatic duct, portal vein and hepatic arteries. In this study, percutaneous electrode placement will be performed under CT fluoroscopy guidance with transcatheter arteriography to prevent puncture of major vessels or electrode placement too close to vital structures. During intraoperative sessions, inspection of the hilum and the use of US will guide electrode placement. Severe complications associated with the procedure may include bile leakage and portal vein or hepatic artery thrombosis, necessitating careful monitoring and instruction of patients on discharge. Specific attention will be paid to the placement of electrodes close to bile ducts, as it was previously demonstrated that biliary strictures may occur when needles are placed within $2-3 \mathrm{~mm}$ of the bile ducts. ${ }^{22}{ }^{37}$ IRE will not be applied in the event of complete or partial thrombosis of the main portal vein. Partial portal vein thrombosis is considered an exclusion criterion because of the potential risk of worsening of the thrombus, leading to sudden complete portal vein thrombosis in such patients. To reduce the risk of cardiac arrhythmias, patients with a history of severe cardiac disease are not considered eligible for this study. Also, cardiac synchronisation during IRE ablation (ie, pulses not given during the atrial or ventricular systole periods of the cardiac cycle) will be applied to further lower the risk of arrhythmias. ${ }^{38} 39$

In the present study, a rate of severe complications of $60 \%$ within 90 days after IRE is considered an acceptable upper limit. Complications in the standard palliative treatment of patients with unresectable PHC are mainly caused by biliary stent dysfunctions. Dislocation, migration or clogging of stents causes cholangitis with or without a rise in plasma bilirubin, requiring reintervention. This is often accompanied by sepsis and/or liver abscesses. In a prospective study including 157 patients with malignant hilar biliary obstruction, $23 \%$ early $(<30$ days) and $40 \%$ late ( $>30$ days) drainage-related complications were observed. ${ }^{33}$ It is essential to evaluate complications in the present study in light of the association with the IRE procedure itself. However, the maximum accepted complication rate consists of both IRE-related and IRE-unrelated complications. To limit the number of drainage procedures, an expert panel has been initiated for this study to carefully assess the individual biliary drainage strategy.

Although the ALPACA study is primarily designed to assess the safety and feasibility of IRE, our secondary hypothesis is that IRE will prolong OS for 3 months. Patients may also benefit from longer metal stent patency, as was previously reported after PDT in advanced PHC. ${ }^{9}$ Despite the higher OS benefit claimed in the treatment of other HPB tumours in current literature, we chose 3 months as this reflects, in our perspective, the least clinically relevant survival benefit for an emerging treatment provided that the complication rate is acceptable. Given the primary study purpose, a control group of patients only receiving standard systemic chemotherapy is not included as a treatment arm. However, long-term outcome in patients undergoing IRE and systemic chemotherapy will be compared with patients who were eligible for study participation but preferred standard palliative 
treatment. Naturally, a valid comparison between IRE and other local therapies or standard treatment may only be possible in a future well-designed prospective randomised controlled trial.

\section{Author affiliations}

${ }^{1}$ Department of Surgery, Academic Medical Center, Amsterdam, The Netherlands

${ }^{2}$ Department of Radiology, VU University Medical Center, Amsterdam, The

Netherlands

${ }^{3}$ Department of Radiology, Academic Medical Center, Amsterdam, The Netherlands ${ }^{4}$ Department of Gastroenterology, VU University Medical Center, Amsterdam, The Netherlands

${ }^{5}$ Department of Gastroenterology, Academic Medical Center, Amsterdam, The Netherlands

${ }^{6}$ Department of Surgery, VU University Medical Center, Amsterdam, The Netherlands ${ }^{7}$ Department of Medical Oncology, Academic Medical Center, Amsterdam, The Netherlands

${ }^{8}$ Department of Medical Oncology, VU University Medical Center, Amsterdam, The Netherlands

Collaborators Trial steering committee: Department of Surgery: ER, GK, TMvG Department of Radiology: LGPHV, KPvL, MRM; Department of Gastroenterology: JEvH, FvDt; Department of Medical Oncology: H-JK, HMV. ALPACA expert panel: Department of Surgery: MGB, ORCB, GK, TMvGu; Department of Radiology: KPvL, OMvD, MRM; Department of Gastroenterology: JEvH, EAJR, FvD.

Contributors RJSC, JAV, LGPHV: trial conception and design, drafting and revising the manuscript. ER, ORCB, OMvD, FvD, JEvH, GK, H-JK, KPvL, EAJR, HMV: trial design, revising the manuscript. MH, HJS, JdV, JWW, BMZ: assisting trial design and revising of the manuscript. MGB: trial conception and design, supervising the drafting and revising of the manuscript. TMvG and MRM: principal investigators, trial conception and design, supervising the drafting and revising of the manuscript. All authors read and approved the final manuscript.

Funding The NanoKnife electrodes that will be used in this study are provided by AngioDynamics (Amsterdam-Zuidoost, the Netherlands). The study protocol has not undergone peer review by a funding body.

Competing interests MRM and KPvL are paid educational consultants for AngioDynamics.

Ethics approval The Institutional Review Board (IRB) of the Academic Medical Center in Amsterdam approved the protocol on 19 April 2016. The study has been registered with the Dutch Central Committee on Research Involving Human Subjects (NL56231.018.15). Secondary approval was provided by the local IRB at VU University Medical Center on 14 July 2016.

Provenance and peer review Not commissioned; externally peer reviewed.

Open Access This is an Open Access article distributed in accordance with the Creative Commons Attribution Non Commercial (CC BY-NC 4.0) license, which permits others to distribute, remix, adapt, build upon this work non-commercially, and license their derivative works on different terms, provided the original work is properly cited and the use is non-commercial. See: http://creativecommons.org/ licenses/by-nc/4.0/

(c) Article author(s) (or their employer(s) unless otherwise stated in the text of the article) 2017. All rights reserved. No commercial use is permitted unless otherwise expressly granted.

\section{REFERENCES}

1. Edge SB BD, Compton CC, Fritz AG, eds. AJCC cancer staging manual. 7th ed. New York: NY: Springer, 2010.

2. van der Geest LA. Kankerzorg in beeld. Galblaas- en proximaal galwegcarcinoom. Rapportage IKNL 2014.

3. Popescu I, Dumitrascu T. Curative-intent surgery for hilar cholangiocarcinoma: prognostic factors for clinical decision making. Langenbecks Arch Surg 2014;399:693-705.

4. van Gulik TM, Kloek JJ, Ruys AT, et al. Multidisciplinary management of hilar cholangiocarcinoma (Klatskin tumor): extended resection is associated with improved survival. Eur J Surg Oncol 2011;37:65-71.

5. Groot Koerkamp B, Wiggers JK, Gonen M, et al. Survival after resection of perihilar cholangiocarcinoma-development and external validation of a prognostic nomogram. Ann Oncol 2016;27:753.
6. Ruys AT, van Haelst S, Busch OR, et al. Long-term survival in hilar cholangiocarcinoma also possible in unresectable patients. World $J$ Surg 2012;36:2179-86.

7. Matsuo K, Rocha FG, Ito K, et al. The Blumgart preoperative staging system for hilar cholangiocarcinoma: analysis of resectability and outcomes in 380 patients. J Am Coll Surg 2012;215:343-55.

8. Valle J, Wasan $\mathrm{H}$, Palmer $\mathrm{DH}$, et al. Cisplatin plus gemcitabine versus gemcitabine for biliary tract Cancer. N Engl J Med 2010;362:1273-81.

9. Cheon YK, Lee TY, Lee SM, et al. Longterm outcome of photodynamic therapy compared with biliary stenting alone in patients with advanced hilar cholangiocarcinoma. HPB 2012;14:185-93.

10. Polistina FA, Guglielmi R, Baiocchi $C$, et al. Chemoradiation treatment with gemcitabine plus stereotactic body radiotherapy for unresectable, non-metastatic, locally advanced hilar cholangiocarcinoma. results of a five year experience. Radiother Oncol 2011;99:120-3.

11. Mahadevan A, Dagoglu N, Mancias J, et al. Stereotactic body radiotherapy (SBRT) for intrahepatic and hilar cholangiocarcinoma. $J$ Cancer 2015;6:1099-104.

12. Wagner A, Denzer UW, Neureiter D, et al. Temoporfin improves efficacy of photodynamic therapy in advanced biliary tract carcinoma: a multicenter prospective phase II study. Hepatology 2015;62:1456-65.

13. Ortner ME, Caca K, Berr F, et al. Successful photodynamic therapy for nonresectable cholangiocarcinoma: a randomized prospective study. Gastroenterology 2003;125:1355-63.

14. Dolak W, Schreiber F, Schwaighofer $\mathrm{H}$, et al. Endoscopic radiofrequency ablation for malignant biliary obstruction: a nationwide retrospective study of 84 consecutive applications. Surg Endosc 2014;28:854-60.

15. Laquière $A$, Boustière $C$, Leblanc $S$, et al. Safety and feasibility of endoscopic biliary radiofrequency ablation treatment of extrahepatic cholangiocarcinoma. Surg Endosc 2016;30:1242-8.

16. Goldberg SN, Hahn PF, Tanabe KK, et al. Percutaneous radiofrequency tissue ablation: does perfusion-mediated tissue cooling limit coagulation necrosis? J Vasc Interv Radiol 1998;9:101-11.

17. Delemotte L, Tarek M. Molecular dynamics simulations of lipid membrane electroporation. J Membr Biol 2012;245:531-43.

18. Tarek M. Membrane electroporation: a molecular dynamics simulation. Biophys J 2005;88:4045-53.

19. Weaver JC, Vaughan TE, Chizmadzhev Y. Theory of electrical creation of aqueous pathways across skin transport barriers. Adv Drug Deliv Rev 1999;35:21-39.

20. Narayanan G, Bhatia S, Echenique A, et al. Vessel patency post irreversible electroporation. Cardiovasc Intervent Radiol 2014;37:1523-9.

21. Kingham TP, Karkar AM, D'Angelica MI, et al. Ablation of perivascular hepatic malignant tumors with irreversible electroporation. J Am Coll Surg 2012;215:379-87.

22. Choi JW, Lu DS, Osuagwu F, et al. Assessment of chronological effects of irreversible electroporation on hilar bile ducts in a porcine model. Cardiovasc Intervent Radiol 2014;37:224-30.

23. Melenhorst MC, Scheffer HJ, Vroomen LG, et al. Percutaneous irreversible electroporation of unresectable hilar cholangiocarcinoma (Klatskin Tumor): A Case Report. Cardiovasc Intervent Radiol 2016;39:117-21.

24. Cannon R, Ellis S, Hayes D, et al. Safety and early efficacy of irreversible electroporation for hepatic tumors in proximity to vital structures. J Surg Oncol 2013;107:544-9.

25. Cheung W, Kavnoudias H, Roberts S, et al. Irreversible electroporation for unresectable hepatocellular carcinoma: initial experience and review of safety and outcomes. Technol Cancer Res Treat 2013;12:233-41

26. Martin RC, McFarland K, Ellis S, et al. Irreversible electroporation in locally advanced pancreatic cancer: potential improved overall survival. Ann Surg Oncol 2013;20:443-9.

27. Rombouts SJ, Vogel JA, van Santvoort HC, et al. Systematic review of innovative ablative therapies for the treatment of locally advanced pancreatic Cancer. Br J Surg 2015;102:182-93.

28. Scheffer HJ, Nielsen K, de Jong MC, et al. Irreversible electroporation for nonthermal tumor ablation in the clinical setting: a systematic review of safety and efficacy. J Vasc Interv Radiol 2014;25:997-1011.

29. Belfiore MP, Ronza FM, Romano F, et al. Percutaneous CT-guided irreversible electroporation followed by chemotherapy as a novel neoadjuvant protocol in locally advanced pancreatic cancer: Our preliminary experience. Int J Surg 2015;21:S34-S39. 
30. Narayanan G, Hosein PJ, Arora G, et al. Percutaneous irreversible electroporation for downstaging and control of unresectable pancreatic adenocarcinoma. J Vasc Interv Radiol 2012;23:1613-21.

31. Silk MT, Wimmer T, Lee KS, et al. Percutaneous ablation of peribiliary tumors with irreversible electroporation. J Vasc Interv Radiol 2014;25:112-8.

32. Belfiore G, Belfiore MP, Reginelli A, et al. Concurrent chemotherapy alone versus irreversible electroporation followed by chemotherapy on survival in patients with locally advanced pancreatic Cancer. Med Oncol 2017;34:38.

33. De Palma GD, Galloro G, Siciliano S, et al. Unilateral versus bilateral endoscopic hepatic duct drainage in patients with malignant hilar biliary obstruction: results of a prospective, randomized, and controlled study. Gastrointest Endosc 2001;53:547-53.

34. Scheffer HJ, Vogel JA, van den Bos W, et al. The influence of a metal stent on the distribution of thermal energy during irreversible electroporation. PLoS One 2016;11:e0148457.
35. Narayanan G, Hosein PJ, Beulaygue IC, et al. Percutaneous ImageGuided irreversible electroporation for the treatment of Unresectable, locally Advanced pancreatic adenocarcinoma. J Vasc Interv Radiol 2017;28:342-8.

36. Wiggers JK, Groot Koerkamp B, Coelen RJ, et al. Preoperative biliary drainage in perihilar cholangiocarcinoma: identifying patients who require percutaneous drainage after failed endoscopic drainage. Endoscopy 2015;47:1124-31.

37. Lu DS, Kee ST, Lee EW, et al. Irreversible electroporation: ready for prime time? Tech Vasc Interv Radiol 2013;16:277-86.

38. Deodhar A, Dickfeld T, Single GW, et al. Irreversible electroporation near the heart: ventricular arrhythmias can be prevented with ECG synchronization. AJR Am J Roentgenol 2011;196:W330-W335.

39. Thomson KR, Cheung W, Ellis SJ, et al. Investigation of the safety of irreversible electroporation in humans. $J$ Vasc Interv Radiol 2011;22:611-21. 